The Application Of Mid-Ranging Control To Improve Thermal Disturbance Rejection for Cryogenic THD/DT Layering At The National Ignition Facility

M. Dayton, B. Haid

June 4, 2013

25th Symposium on Fusion Engineering

San Francisco, CA, United States

June 10, 2013 through June 14, 2013 
This document was prepared as an account of work sponsored by an agency of the United States government. Neither the United States government nor Lawrence Livermore National Security, LLC, nor any of their employees makes any warranty, expressed or implied, or assumes any legal liability or responsibility for the accuracy, completeness, or usefulness of any information, apparatus, product, or process disclosed, or represents that its use would not infringe privately owned rights. Reference herein to any specific commercial product, process, or service by trade name, trademark, manufacturer, or otherwise does not necessarily constitute or imply its endorsement, recommendation, or favoring by the United States government or Lawrence Livermore National Security, LLC. The views and opinions of authors expressed herein do not necessarily state or reflect those of the United States government or Lawrence Livermore National Security, LLC, and shall not be used for advertising or product endorsement purposes. 


\title{
The Application of Mid-Ranging Control to Improve Thermal Disturbance Rejection for Cryogenic THD/DT Layering at the National Ignition Facility
}

\author{
M.S. Dayton, B.J. Haid \\ National Ignition Facility \\ Engineering and Controls \\ Livermore, CA 94550 \\ Email: dayton5@1ln1.gov, haid1@1lnl.gov
}

\begin{abstract}
This paper presents a cryogenic temperature control technique that demonstrated good disturbance rejection at the National Ignition Facility. Temperature excursions must be minimized to maintain DT fuel layer symmetry needed for ignition. The control scheme known as mid-ranging control effectively used two manipulated inputs and one output, which differs from traditional single-input-multiple-output control schemes like cascade control. Each input had different power constraints and substantially different dynamic effects on the output. One input acted as a bulk heat source whereas the other acted as a trimming heat source. During an upset, the controller manipulated both input variables simultaneously to maintain the desired temperature. The mid-ranging controller was tuned using an extension of the well-known Ziegler-Nichols $(\mathrm{ZN})$ method. The derived SIMC tuning rules were not as attractive since, to be applied, they required prior knowledge of the target's thermal dynamics. The resulting control scheme rejected large and sudden increases in thermal loading quicker than a more conventional scheme. The technique and tuning equations may be applied to similar cryogenic control problems.
\end{abstract}

Keywords-mid-ranging controller; PID control; cryogenic; DT layering; tuning equations;

\section{INTRODUCTION}

NIF performs a wide range of experiments involving a variety of target configurations. Many of these targets are fielded at cryogenic temperatures and must be precisely controlled to provide the desired conditions within the target at shot-time. Indirect drive ignition targets currently have the most stringent temperature control requirements and must remain stable to within two millikelvins for several days.

Ultimately, there are two main focuses of the cryogenic design. The first is to provide precise and stable temperature control for layer uniformity. The second is to maintain temperature stability against variable thermal loads up to the instant of the laser shot.

The mid-ranging control scheme suits both design objectives [1]. The scheme effectively uses two input variables and one output. One of the input acts as a bulk heater whereas the others act as a trimming heater. During an upset, the controller manipulates both input variables simultaneously to maintain the desired temperature.

We compare the closed-loop disturbance rejection performance of a simple PID control scheme with the performance of the mid-ranging control scheme using the the integrated absolute error (IAE) of the control error $e=y-y r$. For ignition targets, the mid-range scheme responds better to relatively high-magnitude, long-duration disturbances that occur near shot time.

\section{BACKGROUND}

Layering targets contain a frozen deuterium-tritium (DT) fuel mix to be driven to nuclear fusion by a 2 megajoule laser pulse. The fuel is maintained a few hundred millikelvins below the triple point of the mix, at approximately $19 \mathrm{~K}$. Figure 1 illustrates the target assembly that contains the solid DT fuel mix. The assembly includes a copper base that attaches to a cold finger of a cryostat and is cooled by conduction. The cryostat cools the copper base using a Gifford-McMahon (GM) cryocooler.

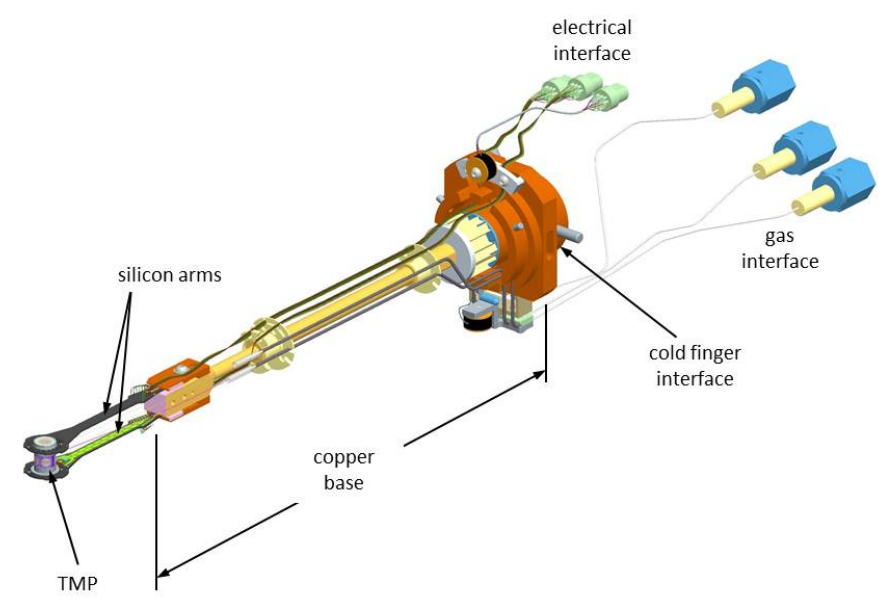

Fig. 1. Overview of the target assembly.

After cooling the assembly below the boiling point of the DT fuel, the fuel is introduced via a capillary into a $2 \mathrm{~mm}$ diameter spherical capsule. The capsule is suspended in the thermal mechanical package (TMP) between a pair of thin plastic films called tents, shown in figure 2. The TMP is an aluminum cylinder $5 \mathrm{~mm}$ diameter by $12 \mathrm{~mm}$ in height. The space between the capsule and the inner wall of the TMP is filled with a gas, typically helium, that conducts heat from 
the capsule to the TMP. Silicon arms provide a cooling path between the TMP and the copper base.

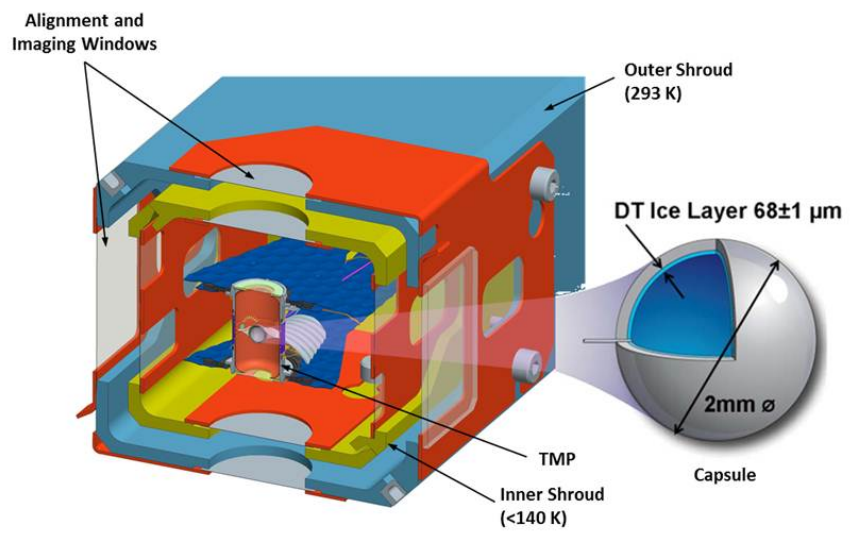

Fig. 2. Detailed cross-section of the TMP and the shroud

The DT fuel is shaped into a uniform layer on the inner surface of the capsule. The process by which the layer is shaped is called beta layering [2]. In beta layering, the layer thickness responds to the temperature at the layers outer surface. If the outer DT surface has uniform temperature and is perfectly spherical, the layer will naturally form with uniform thickness. The TMP's longitudinal temperature profile is adjusted using two shim heaters which are wire wound around the TMP circumference, $2 \mathrm{~mm}$ above and below the silicon arm interfaces. The shims affect the second mode symmetry of the DT layer and are adjusted in response to the shape of the layer. The DT layer shape is revealed by x-ray imaging of the capsule [3]. The shim heaters are not actively controlled.

During a laser shot, the 192 laser beams enter through holes at the end faces of the TMP, referred to as laser entry holes (LEH). The laser is directed onto the inner wall of the hohlraum that is lined with a high- $Z$ material to efficiently convert the incident laser light into x-rays. The $\mathrm{x}$-rays in turn ablate the outer capsule material to cause a compressive force that implodes the DT fuel. The fuel requires a high degree of layer uniformity to implode symmetrically and reach thermonuclear burn. In addition to layer uniformity, the temperatures of the matter contained within the capsule and the TMP in the seconds leading up to the laser shot are critical to the experiments. This statement applies to ignition targets as well as cryogenic targets for other applications.

\section{THERMAL DistURBANCES}

The target is fielded within a vacuum vessel with other systems, some of which generate substantial but variable and nonuniform radiative heat loads. As the ice layer shape is easily affected by changes in the temperature profile across the TMP, it is important that the heat loads from the ambient environment be minimized for layered targets. The general design strategy has been to reduce all external radiative loading such that the TMP heaters determine the temperature distribution. Therefore, the target is enclosed inside a retractable shroud to block the radiative loads from other systems and the ambient environment. The radiative load from the shrouds is reduced by gold-plating the inner surface to lower the emissivity, and by cooling the inner shroud below $140 \mathrm{~K}$. The reduced shroud temperature is essential because, although a room-temperature shroud may block radiation from external sources, it will still emit substantial radiation that affects the TMP's temperature profile.

A large disturbance is introduced when the shrouds are opened eight seconds before the laser shot to expose the target to the laser. The opening causes an approximate $200 \mathrm{~mW}$ step increase in radiative loading from the ambient environment. Additionally for ignition targets, the TMP temperature is ramped down over the last 30 seconds before the shot to reduce the pressure of the DT vapor that is enclosed by the solid layer. For ignition targets, the mid-range scheme provides the greatest advantage in response to these relatively highmagnitude, long-duration disturbances.

\section{A Simplified MOdEL}

The first step in the study and design of the mid-ranging controller was to develop a simplified model of the target's thermal dynamics. Because the target operated in a vacuum, conductive heating dominated the transfer mechanism and helped keep the model simple. In figure 3, the voltage controlled resistive heaters injected power into both silicon arms and at the base of the target. The heat flowed towards the helium cryostat connected at the base of the target.

The thermal response from the arm heaters to the temperature sensor, located near the hohlraum, was approximately 10 times faster than the base heater. The target's arm heaters behaved as trimming heaters for fine adjustment, whereas the target's base heater behaved as a bulk heater.

Both heaters operated below a fixed output power because of hardware constraints, which meant that without the base heater, the arm heaters could not reach the normal operating temperatures. The base heater was able to produce several watts of power compared with the $150 \mathrm{~mW}$ each arm heater could produce. The non-linear constraints were lumped into the functions $f_{1}()$ and $f_{2}()$, seen in figure 3 . Because of the target's symmetry, the model's arm heaters were simplified to a single input, $u(s)$. Consistent target fabrication techniques also ensured that the functions were approximately equal.

Finally, a series of step response experiments at the targets nominal operating point identified the transfer functions for the target's thermal process, equations 1 and 2 . The simplified target process model was illustrated in figure 4 and figure 5 .

where,

$$
\begin{aligned}
& g_{1}(s)=\frac{k_{1}}{\tau_{1} s+1} e^{-\theta_{1} s}=\frac{0.87}{1.08 s+1} e^{-0.14 s} \\
& g_{2}(s)=\frac{k_{2}}{\tau_{2} s+1} e^{-\theta_{2} s}=\frac{4.7}{10.8 s+1} e^{-0.6 s}
\end{aligned}
$$

The controllers took the ideal-form,

$$
\begin{gathered}
c_{1}(s)=k_{p_{1}}\left(1+\frac{1}{\tau_{i_{1}} s}+s \tau_{d_{1}}\right)=11\left(1+\frac{3.9}{s}+s 0.066\right) \\
c_{2}(s)=k_{p_{2}}\left(1+\frac{1}{\tau_{i_{2}} s}\right)
\end{gathered}
$$




\section{PROPOSED APPROACH}

A conventional approach uses a single PID controller for the arm heaters and fix the base heater's input power, seen in figure 4 . The base heater would then need manual adjustments to accommodate a large change in the load conditions. Unfortunately, this approach would fail to stay in control during large load disturbances like a shroud opening. Nevertheless, the simple control scheme was used to provide a baseline for comparison. Other typical control schemes, like cascade control, did not suit the target's dynamics since the inner-loop would be much slower than the outer-loop [4].

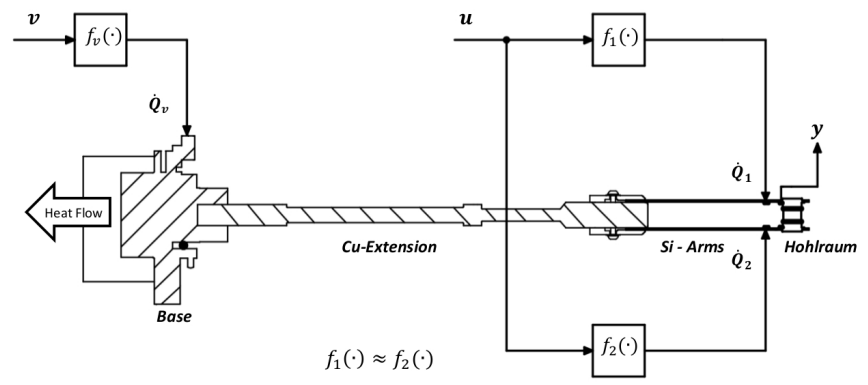

Fig. 3. Illustration of the inputs and controlled variables for a THD target.

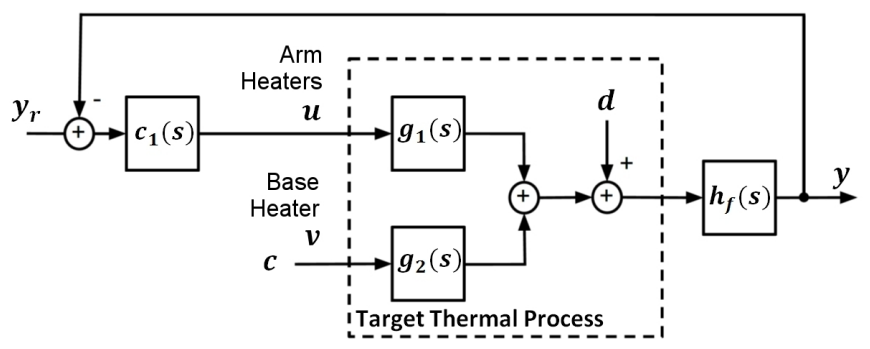

Fig. 4. A Simple PID control scheme for a THD target

We proposed to use a mid-ranging controller. The addition of the controller, seen in figure 5, provides two improvements. One, the controller would automatically adjust the base heater's setpoint during load disturbances, and two, the controller would hold the arm heater's input near their linearized operating voltages, which would help maintain the controller's performance over a wider range of heat loads. In our case Joule heating non-linearities were unavoidable. However, for other applications a square-rooter at the heaters' inputs would remove them.

The sensitivity function describes a control system's closed loop frequency response to load disturbances and the function's peak provides a measure of robustness [5]. A more robust system will have a lower peak. Ideally, the control system would dampen all disturbances, hence the sensitivity function's amplitude would be low for all frequencies. Unfortunately, retuning the controller to dampen out unwanted frequencies often inadvertently amplifies other frequencies.

A comparison of the two control scheme's sensitivity functions, seen in figure 6 , predicted that the benefits of the mid-ranging controller would not come at the cost of robustness or an increase in higher frequency sensitivity. Since the calculations were based on a simplified model, their predictive powers were limited. Nevertheless, they did provide insight into the effects a mid-ranging controller can have on a control system.

The sensitivity function is described in equation 5, where $L(j f)$ is the system's open-loop gain.

$$
|S(j f)|=\frac{1}{|1+L(j f)|}
$$

\section{TUNING STRATEGY FOR THE MID-RANGING CONTROLLER}

A mid-ranging controller was not as straightforward to tune as the simple PID loop. We considered two tuning strategies. The first one was a design approach based on SIMC PI-settings since the tuning equations were simple and general enough to be applied to various types of targets. The SIMC tuning rules were also shown to demonstrate good robustness and disturbance rejection for similar system dynamics [5].

The second approach was based on Ziegler-Nichols (ZN) PID closed loop tuning procedure [6]. The rules were simple enough to be applied to various types of targets and the designer's primary objective was to reduce load disturbances. Typically, the method provides aggressive tunes with good disturbance response for an integrating process [4].

\section{SIMC tuning rules for the mid-ranging controller}

We reduced our model to a first-order approximation using Skrogestads rules [5] and derived SIMC tuning parameters by following similar arguments Bruce Allision and Shiro Ogawa make [7].

First, while the mid-ranging controller was set to zero, the simple-PID controller $c_{1}(s)$ was tuned for disturbance rejection. Without the mid-ranging controller, the simple-PID closed-loop response was approximated to a single order transfer function, where $\tau_{y}$ was equal to the closed-loop time constant. Since the dynamics of $g_{1}(s)$ were much faster than $g_{2}(s)$, the two loops did not strongly interact.

$$
G_{y}(s)=\frac{y(s)}{y_{r}(s)}=\frac{c 1(s) g_{1}(s) h_{f}(s)}{1+c_{1}(s) g_{1}(s) h_{f}(s)} \approx \frac{1}{1+\tau_{y} s}
$$

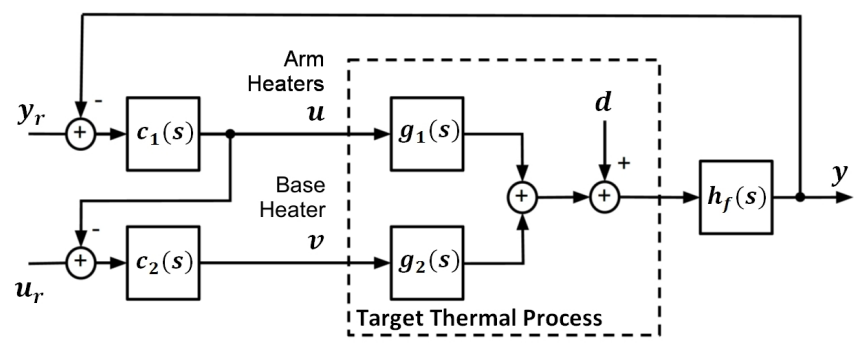

Fig. 5. A Simple PID control scheme with the addition of a mid-ranging controller $c_{2}(s)$ for a THD target 


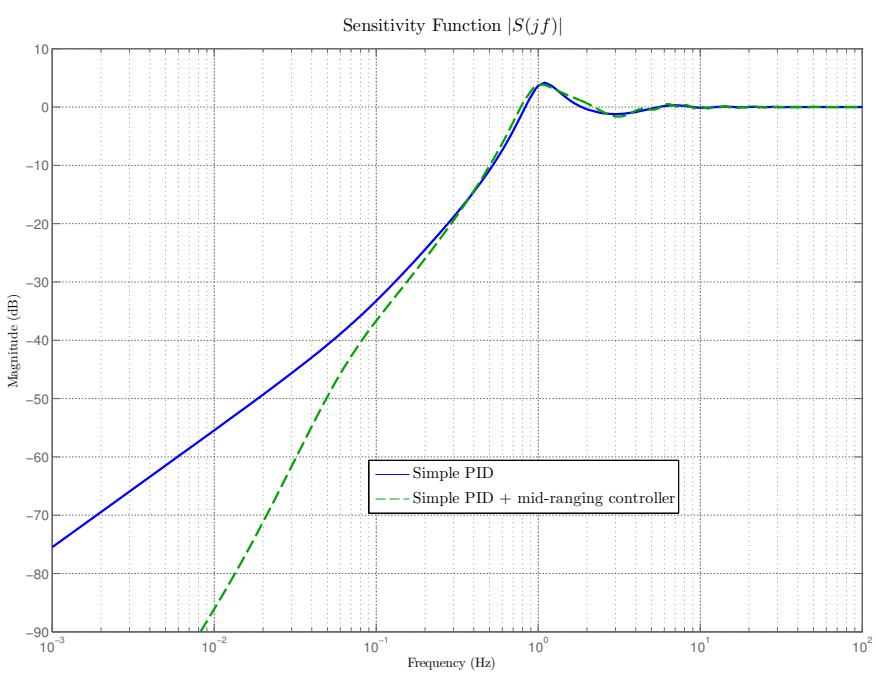

Fig. 6. The calculated sensitivity function for both control schemes.

Recall that the mid-ranging controller manipulates the input variable $v(s)$ until the other input variable $u(s)$ reaches the controller's setpoint. The next step was to derive the openloop system dynamics for the mid-ranging PI controller $c_{2}(s)$.

$$
g_{v}=\frac{u(s)}{v(s)}=-\frac{g_{2}(s) c_{1}(s) h_{f}(s)}{1+c_{1}(s) g_{1}(s) h_{f}(s)}=-\frac{g_{2}(s)}{g_{1}(s)} G_{y}(s)
$$

The system's first order approximations were substituted in equation 7.

$$
g_{v}=-\frac{k_{2}}{k_{1}} \frac{\left(\tau_{1} s+1\right)}{\left(\tau_{2} s+1\right)} \frac{e^{-s \theta}}{\left(\tau_{y} s+1\right)}: \theta=\theta_{2}+\theta_{y}-\theta_{1}
$$

The dynamics in equation 8 were still too complicated for a PI controller. According to the analytic rules proposed in reference [5], the equation may be reduced to a single order transfer function.

$$
g_{v} \approx-\frac{k_{2}}{k_{1}} \frac{5 \theta^{\prime}}{\tau_{2}} \frac{e^{-s \theta^{\prime}}}{\left(\tau_{v} s+1\right)}: \tau_{v}=5 \theta^{\prime}-\tau_{1}+\frac{\tau_{y}}{2}: \theta^{\prime}=\theta+\frac{\tau_{y}}{2}
$$

given that,

$$
\tau_{1} \leq 5 \theta^{\prime} \leq \tau_{2}: \tau_{y} \ll \tau_{1}
$$

The derived SIMC tuning rules for a PI mid-ranging controller were in the ideal-form, equation 11.

$$
k_{p_{2}}=-\frac{0.5}{k} \frac{\tau_{v}}{\theta^{\prime}}, \tau_{i_{2}}=\tau_{v}: k=-\frac{k_{2}}{k_{1}} \frac{5 \theta^{\prime}}{\tau_{2}}
$$

TABLE I. Mid-RANGing (MR) PI CONTROLleR SETtingS AND PERFORMANCE SUMMARY

\begin{tabular}{l|ccc} 
& \multicolumn{3}{|c}{ Control Scheme : Tuning Method } \\
\hline MR Controller Parameters & MR : ZN & MR : SIMC & Simple PID : ZN \\
\hline$k_{p 2}$ & -0.9 & -1.04 & \\
$\tau_{i 2}$ & 1.54 & 2.27 & \\
\hline Performance Parameters & & & \\
\hline IAE $^{a}$ & 0.12 & 0.12 & \\
GM & 2.45 & 2.45 & 2.36 \\
Gain crossover freq. (Hz) & 0.87 & 0.90 & 0.87 \\
PM (degrees) & 48.4 & 47.3 & 46.2 \\
Phase crossover freq. (Hz) & 1.46 & 1.46 & 1.45 \\
$|S(j f)|_{\text {max }}$ & 1.59 & 1.59 & 1.6 \\
Sensitivity freq. (Hz) & 1.9 & 1.9 & 1.7 \\
\hline${ }^{a}$ taken from figure 7 & \multicolumn{4}{|}{}
\end{tabular}

\section{Ziegler-Nichols (ZN) PID tuning for mid-ranging controller}

ZN's tuning method requires a slow increase in the controller's P term until the output starts to oscillate without the use of the I and D terms [6]. Since the method cannot use an integral term, the mid-ranging controller's output was offset to hold the input variable $u(s)$ at the nominal operating point. The $\mathrm{P}$ term at which the output began to oscillate was recorded as the ultimate gain $K_{u}$ and the period of the oscillations were recorded as $T_{u}$. The following equations were used to calculate the controller settings.

$$
k_{p_{2}}=\frac{2}{5} K_{u}, \tau_{i_{2}}=\frac{4}{5} T_{u}
$$

\section{EVALUATION OF THE TUNING RULES}

The integrated absolute error (IAE) of the control error $e=y-y r$ was used to compare the closed-loop disturbance rejection performance. Both tuning strategies produced the same IAE during a shroud opening while the setpoint remained constant. We compared their phase margins and gain margins to measure robustness. The results are summarized in Table I. The $\mathrm{ZN}$ method provided a tune that was comparable to the SIMC method. However, the ZN method did not require a simplified mathematical model of the target's dynamics. This was valuable since a tune could be developed rapidly for various types of targets.

\section{COMPARISON OF THE CONTROL SCHEMES}

Two tests demonstrated the disturbance rejection of the mid-ranging and the simple PID controller schemes. The first test compared the IAE during a shroud opening while the setpoint remained steady. The second test compared the IAE while the setpoint was ramped down 1.5 Kelvin over 30 seconds to represent a quench, typically done just before a shot. The second test illustrated the controllable range of both schemes.

The mid-ranging controller maintained control and the temperature fully recovered during the shroud opening. On the contrary, the simple PID controller never regained complete control and thermal runaway started after 25 seconds seen in figure 7. At shot time, the mid-ranging control scheme would have produced 20 percent less IAE than the simple PID control scheme.

During a setpoint ramp and shroud opening, the midranging controller kept the peak temperature excursion during 

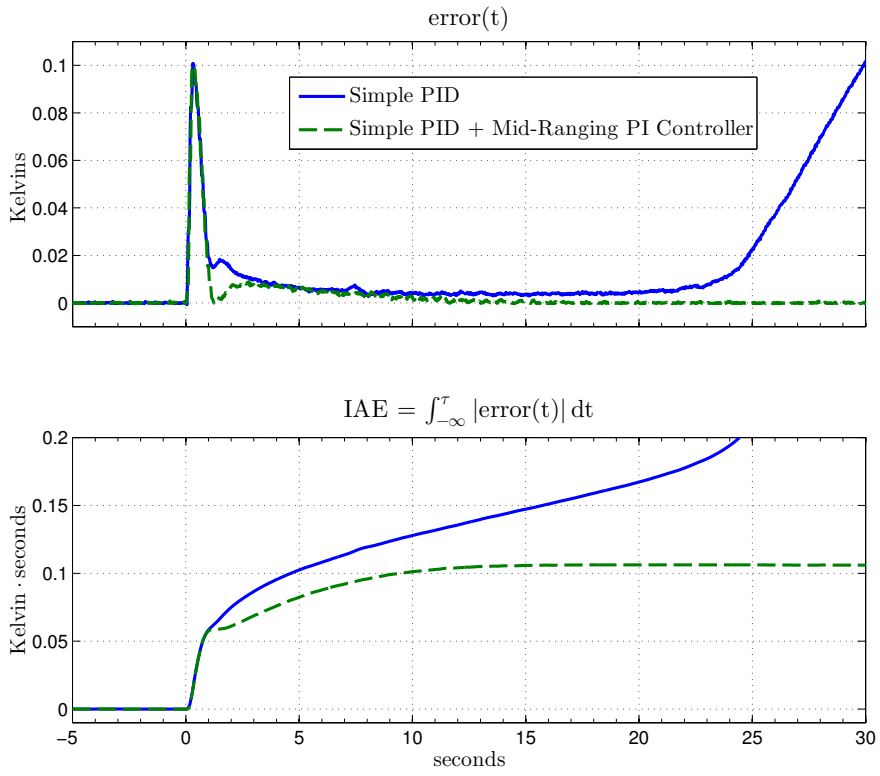

Fig. 7. The IAE comparison for both control schemes during a shroud opening and steady setpoint.
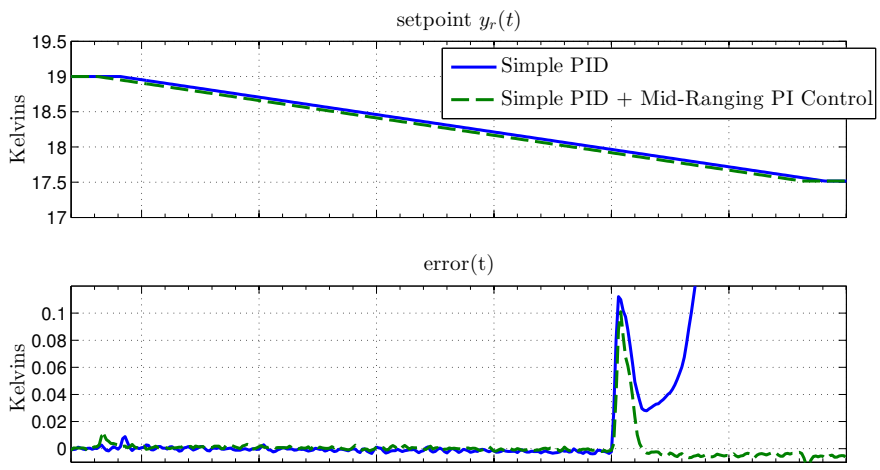

$\operatorname{IAE}=\int_{-\infty}^{\tau}|\operatorname{error}(\mathrm{t})| \mathrm{d} t$

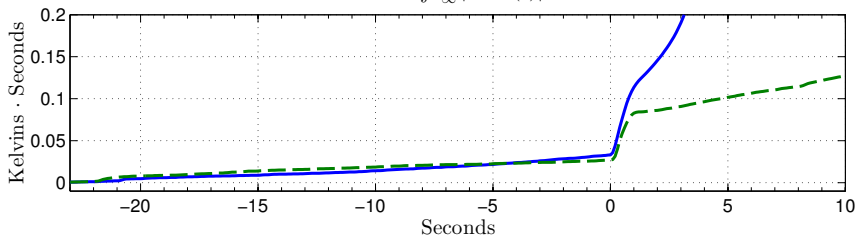

Fig. 8. The IAE comparison for both control schemes during a shroud opening and a typical setpoint ramp that usually proceeds a shot.

the shroud opening slightly lower than the simple PID configuration. More importantly, the mid-ranging controller was able to maintain control after the shroud opening, seen in figure 8 . The test results exemplified the simple PID control scheme's output unreachability caused by input saturation.

\section{CONCLUSION}

We tuned the mid-ranging controller with an extension of the Ziegler-Nichols and SIMC tuning rules. Both methods resulted in the same IAE during a shroud opening. However, the derived SIMC tuning rules were not as attractive as the $\mathrm{ZN}$ method since they could not be applied as rapidly to various types of targets. The mid-ranging control scheme rejected large and sudden increases in thermal loading quicker and with 20 percent less IAE compared with a more conventional scheme. At NIF, the control scheme has worked successfully for 39 layered shots.

\section{ACKNOWLEDGEMENT}

We thank Fred Coffield and Jim Watson for their contributions.

This work was performed under the auspices of the U.S. Department of Energy by Lawrence Livermore National Laboratory under Contract DE-AC52-07NA27344.

\section{REFERENCES}

[1] B. J. Allison and A. J. Isaksson, "Design and performance of mid-ranging controllers," Journal of Process Control, vol. 8, no. 5, pp. 469-474, 1998.

[2] J. K. Hoffer and L. R. Foreman, "Radioactively induced sublimation in solid tritium," Physical Review Letters, vol. 60, no. 13, pp. 1310-1313, 1988.

[3] M. A. Fedorov and et al, "Control system for cryogenic thd/dt layering at the national ignition facility," Proceedings of ICALEPCS2O11, THCHMUST01, 2011

[4] K. J. Astrom, PID controllers: theory, design and tuning. Instrument Society of America, 1995.

[5] S. Skogestad, "Simple analytic rules for model reduction and PID controller tuning," Journal of Process Control, vol. 13, no. 4, pp. 291 309, 2003.

[6] J. Ziegler and N. Nichols, "Optimum settings for automatic controllers," trans. ASME, vol. 64, no. 11, 1942.

[7] B. J. Allison and S. Ogawa, "Design and tuning of valve position controllers with industrial applications," Transactions of the Institute of Measurement and Control, vol. 25, no. 1, pp. 3-16, 2003. 